\title{
A Dual-Modal CT/US Kidney Phantom Model for Image-Guided Percutaneous Renal Access
}

\author{
João Gomes-Fonseca ${ }^{1,2(\bowtie)}$, Alice Miranda ${ }^{1,2}$, Pedro Morais ${ }^{1,2,3,4}$, \\ Sandro Queirós ${ }^{1,2,4,5}$, António C.M. Pinho ${ }^{6}$, Jaime C. Fonseca ${ }^{5}$, \\ Jorge Correia-Pinto $^{1,2,7}$, Estêvão Lima ${ }^{1,2,8}$, and João L. Vilaça ${ }^{1,2,9}$ \\ ${ }^{1}$ School of Medicine, Life and Health Sciences Research Institute (ICVS), \\ University of Minho, Braga, Portugal \\ joaofonseca@med.uminho.pt \\ 2 ICVS/3B's-PT, Government Associate Laboratory, \\ Braga, Guimarães, Portugal \\ ${ }^{3}$ Faculdade de Engenharia, Instituto de Ciência e Inovação em Engenharia \\ Mecânica e Engenharia Industrial, Universidade do Porto, Porto, Portugal \\ ${ }^{4}$ Lab on Cardiovascular Imaging, and Dynamics, Department of Cardiovascular \\ Sciences, KULeuven - University of Leuven, Leuven, Belgium \\ 5 Algoritmi Center, School of Engineering, University of Minho, \\ Guimarães, Portugal \\ ${ }^{6}$ Department of Mechanical Engineering, School of Engineering, \\ University of Minho, Guimarães, Portugal \\ 7 Department of Pediatric Surgery, Hospital of Braga, Braga, Portugal \\ ${ }^{8}$ Department of CUF Urology, Hospital of Braga, Braga, Portugal \\ ${ }^{9}$ DIGARC-Technology School, Polytechnic Institute of Cávado and Ave, \\ Barcelos, Portugal
}

\begin{abstract}
Percutaneous renal access (PRA) is a crucial step in some minimally invasive kidney interventions. During this step, the surgeon inserts a needle through the skin until the kidney target site using fluoroscopy and ultrasound imaging. Recently, new concepts of enhanced image-guided interventions have been introduced in these interventions. However, their validation remains a challenging task. Phantom models have been presented to solve such challenge, using realistic anatomies in a controlled environment. In this work, we evaluate the accuracy of a porcine kidney phantom for validation of novel dual-modal computed tomography (CT)/ultrasound (US) image-guided strategies for PRA. A porcine kidney was combined with a tissue mimicking material (TMM) and implanted fiducial markers (FM). While the TMM mimics the surrounding tissues, the FM are used to accurately assess the registration errors between the US and CT images, providing a valid ground-truth. US and CT image acquisitions of the phantom model were performed and the FM were manually selected on both images. A rigid alignment was performed between the selected FM, presenting a root-mean-square error of $1.1 \mathrm{~mm}$. Moreover, the kidney was manually segmented, presenting volumes of $203 \mathrm{ml}$ and $238 \mathrm{ml}$ for CT and US, respectively. The initial results are promising on achieving a realistic kidney phantom model to develop new strategies for PRA, but further work to improve the manufacturing process and to introduce motion and anatomical artifacts in the phantom is still required.
\end{abstract}


Keywords: Image-guided interventions $\cdot$ Kidney $\cdot$ Percutaneous renal access · Porcine phantom

\section{Introduction}

Percutaneous renal access (PRA) plays a key role in minimally invasive kidney interventions (MIKI), such as percutaneous nephrolithotomy, radiofrequency ablation of renal tumors, and kidney biopsies [1]. During the preoperative and intraoperative phases of MIKI that use PRA, they include two important steps: the surgical planning, which relies on the evaluation of richly-detailed pre-operative data, namely computed tomography (CT) and during which the surgeon sets out the strategy of the intervention (e.g., the preferred path to target the surgical site); and, the PRA, where the surgeon inserts a surgical needle from the skin until the kidney target site, guiding the needle position using intraoperative imaging. Fluoroscopy and two-dimensional (2D) ultrasound (US) are the most common imaging modalities for PRA, due to the real-time depiction of the renal system [2]. However, since fluoroscopy exposes both patients and surgeons to a significant amount of radiation, pure US image guidance appears as a potential and attractive solution.

The surgeon's ability to visualize and reach the anatomical target during PRA restricts the MIKI success. The ideal PRA is one that allows an accurate access to the kidney target site minimizing bleeding. Therefore, PRA remains a challenging task [3]. Inaccurate and multiple punctures often cause complications [4], where injuries to the kidney or contiguous organs can eventually prejudice the surgical outcome.

Many paths and technological advances have been proposed to improve the PRA, and, lately, the concepts of enhanced image-guided interventions (eIGI) have been introduced for PRA [5]. eIGI are computer-based systems that overlap different imaging data to improve the physicians' perception of the target site. They intend to aid physicians performing medical procedures with higher accuracy and minimizing the risks. eIGI systems are built based on different concepts as imaging, tracking, segmentation, registration, visualization, and software [6]. Thus, as part of incorporation of enhanced image-guided concepts in PRA, motion tracking of surgical instruments has been studied for PRA [7-10]. Combined with imaging modalities, optical or electromagnetic tracking proved its added-value for PRA. Regarding merely the medical imaging, several authors have explored multimodal imaging for PRA [11, 12]. They fused anatomical information of preoperative images - such as CT and Magnetic Resonance Imaging - with the intraoperative images.

The development and incorporation in the clinical practice of new image-guided systems in PRA are limited by the appropriate validation applied. Currently, phantoms are accepted as gold-standard because they mimic the properties of the tissue in a controlled environment. Moreover, this type of validation is crucial before animal experiments and clinical trials, allowing software tuning and improving.

Studies have been made to build kidney phantom models for the validation of image-guided frameworks. Xiang et al. developed a kidney phantom model based on a kidney porcine and an agar solution [13]. Hunt et al. described the manufacturing of low-cost renal phantoms models based on patient-specific kidney model from CT data 
and gelatin gel material [14]. Likewise, Ristolainen et al. developed a kidney phantom using concepts of three-dimensional (3D) printing and molding to create a kidney model with calyxes. Both gelatin and silicone are applied [15]. Following a similar methodology, Adams et al. presented a soft 3D-printed phantom of the kidney with collecting system [16]. They start by scanning human cadaveric kidneys and constructing phantoms using 3D wax printing and polymer molding.

Despite the current revolution of 3D-printed phantom models, they still have limitations; mainly mimicking the whole characteristics of the different kidney tissues. Thus, the images should be closer to the real ones, encouraging the development of novel eIGI systems. Phantom models of ex-vivo porcine organs have shown successful results in CT and US [13,17, 18], where realistic images are acquired.

Therefore, the aim of this work is to present a methodology to create a CT/US kidney phantom model. The accuracy of this phantom model will be preliminarily evaluated and compared with the expected restrictions of the PRA interventions.

\section{Materials and Methods}

In this section, we describe the protocol used to manufacture the kidney phantom model. This phantom is composed of three main components: a porcine kidney, a tissue mimicking material (TMM), and implanted fiducial markers (FM).

\subsection{Porcine Kidney}

The porcine kidney was obtained from a local slaughterhouse. The renal hilum, ureter, artery, and vein were undamaged. External cleaning was made, where fat tissue and renal capsule were removed. A saline solution was flushed via artery to remove blood. Then, a solution of a contrast agent (Telebrix ${ }^{\circledR} 35$, France) was injected to enhance the kidney on CT images. In detail, $40 \mathrm{ml}$ solution of contrast agent diluted in saline solution (1:20) was injected into the renal artery. Afterward, the renal artery and vein were closed with suture thread.

\subsection{Tissue Mimicking Material}

An agar-based tissue mimicking material (TMM), similar to the one presented by Terlinck et al. [19], was used to enclose the porcine kidney and allow acquisition through US. The composition (weight \%) was: 3\% agar-agar; $11 \%$ glycerol; $83.25 \%$ distilled water; $2.5 \%$ flour; and $0.25 \%$ bleach. This agar percentage made the TMM robust to break or tear, the glycerol was required to adjust the speed of sound to $1540 \mathrm{~m} / \mathrm{s}$, and the flour was applied to enhance acoustic scattering [20]. Finally, bleach prevented the microorganisms growth [13].

Specifically, agar, glycerol and distilled water were mixed in a recipient and heated to $95{ }^{\circ} \mathrm{C}$ for $1 \mathrm{~h}$. The reagents were stirred during the heating process. When the solution was uniform, the flour was added and mixed to prevent large clumps. The TMM was heated another $15 \mathrm{~min}$ and, then, it was allowed to cool at room temperature to $50{ }^{\circ} \mathrm{C}$ with continuous stirring. At the end, the bleach was added. 


\subsection{Fiducial Markers}

Each fiducial marker was a polystyrene sphere with $6 \mathrm{~mm}$ of diameter and with a hole to attach a holder. To locate the FM into specific positions, two supports were designed. The supports intended to: (1) be positioned at the bottom and at the top of a box; (2) give enough space to position the kidney on the box; (3) be easily removed while maintaining the FM on the expected positions; (4) position the FM around the kidney; (5) organize the FM nonlinearly; and (6) spread the FM' distribution [21].

According to these restrictions, bottom and top supports integrated twelve holders each, which were equally spaced $(3 \mathrm{~cm})$ in $x$ and $y$. A different distribution in $z$ was used. Thus, we spread four FM in three different heights $(3,4$ and $5 \mathrm{~cm})$ varying their distribution along the support (Fig. 1). In order to easily identify the kidney orientation in US images, different shapes were applied to the bottom and top FM.

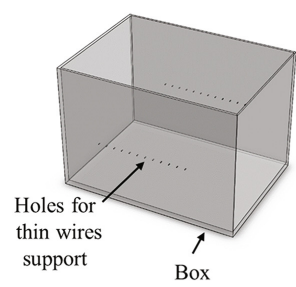

A

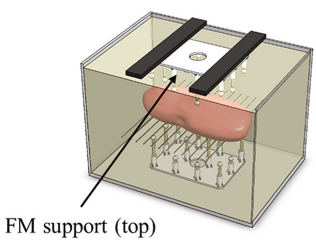

$\mathrm{D}$
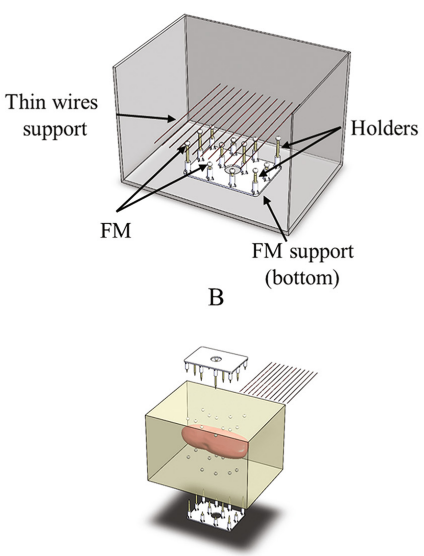

E
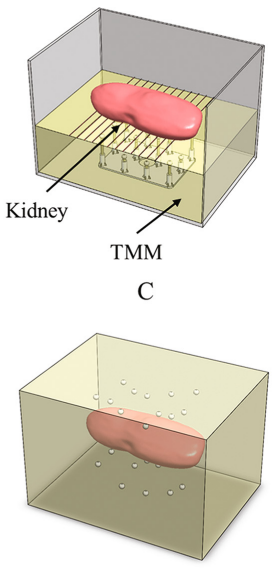

F

Fig. 1. Steps of the phantom's construction process. (A) Box with small holes for thin wires support. (B) Fiducial markers (FM) support is positioned at the bottom and the thin wires in the box holes. (C) First pouring of the tissue mimicking material (TMM). (D) After positioning the kidney, TMM pouring should continue until reaching the box top. (E) During the TMM hardening, the thin wires are removed (after $1 \mathrm{~h}$ ) and, then, the FM supports are also carefully detached (after 8-10 h), F) the FM are fixed at their positions.

\subsection{Phantom Construction}

A box (Fig. 1A) with the following dimensions $16 \times 24 \times 15 \mathrm{~cm}$ was prepared. To enclose the kidney into the TMM and to keep it centered on the box, we used a thin wires support (Fig. 1B). The thin wires support was positioned at $6.5 \mathrm{~cm}$ of the box bottom and the support held the kidney during pouring of the TMM.

The TMM was poured into the box until the thin wires support. Then, the kidney was placed on this support (Fig. 1C) and the pouring process continued until filling the box (Fig. 1D). After cooling for $1 \mathrm{~h}$, the wires were removed. TMM was allowed to 
harden for 8-10 h at room temperature. When the hardening was complete, FM supports were removed (Fig. 1E), without modifying their spatial location (Fig. 1F). Finally, empty spaces were filled with heated TMM.

\section{Experiments}

In this section, we describe how the dual-modal kidney phantom was evaluated.

\subsection{CT and US Images Acquisition}

The CT images were acquired using the Brilliance 64 Scanner (Philips Healthcare, Netherlands). The X-ray tube current was $313 \mathrm{~mA}$ and peak voltage was $120 \mathrm{kV}$, using the abdomen protocol. The image size was $512 \times 512 \times 302$ with a pixel spacing of $0.701 \mathrm{~mm}$, a slice thickness of $2 \mathrm{~mm}$, and a space between slices of $1 \mathrm{~mm}$.

The US images were acquired using the Voluson P6 US system (GE Healthcare, USA). The real-time four-dimensional convex abdominal transducer (RAB2-6-RS, 2-5 MHz) was used. According to the machine settings, 3D images were acquired using high quality, maximum field-of-view and a depth of $15.9 \mathrm{~cm}$. An isotropic volume resolution of $0.667 \mathrm{~mm}$ and an image size of $235 \times 172 \times 197$ was used.

\subsection{Fiducial Registration Error and Volume Differences}

To estimate the fiducial registration error between CT and US volumes, the imageguided therapy toolbox, implemented in 3D Slicer (version 4.6), was used. To manipulate the US volume, the DICOMatic software (Tomovision, Canada) was used to convert the files from the US machine. Initially, each marker was detected on both images. Note that the FM is represented as brighter and darker structures in US and CT images, respectively. The manually detected markers were then aligned based on [22]. The root-mean-square (RMS) was used to assess the alignment error.

To estimate volume differences, one observer performed the manual segmentation of the kidney on both images. The segmentation was performed slice-by-slice along the axis plane. The volume and surface area of each model were compared.

\section{Results}

\subsection{CT and US Images Acquisition}

It was manufactured one phantom using the described protocol. Figure 2 shows the resulting CT and US images from the same phantom. The kidney boundaries and its inner body are visible in both cases. Anatomical details as renal medulla, cortex, and column are detected. Moreover, the results in Fig. 3 prove that the contrast agent is essential to visualize the kidney on the CT images (Fig. 3A-B), while the flour contributes to enhancing the acoustic scattering on US (Fig. 3C-D). 


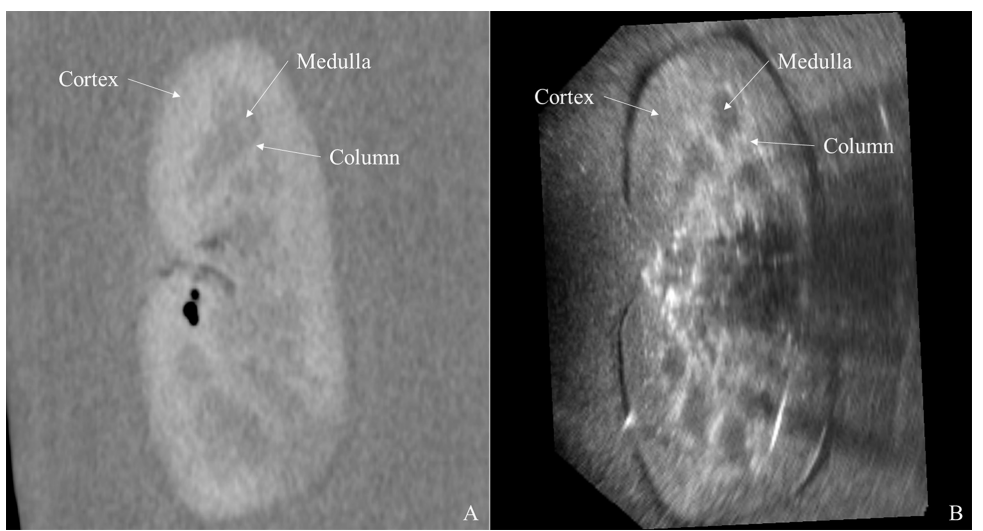

Fig. 2. (A) CT and (B) US images from the same phantom. Kidney boundaries and anatomical details are visible as renal cortex, renal medulla and renal column.
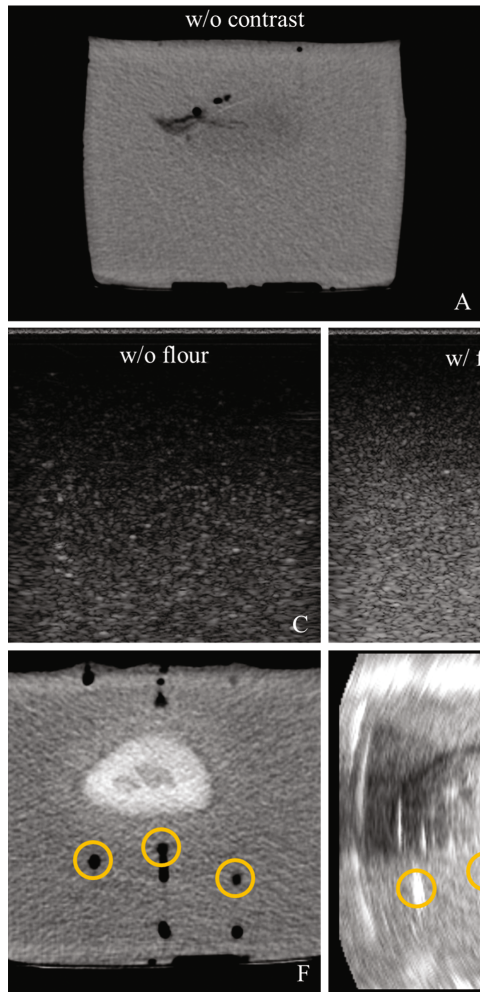
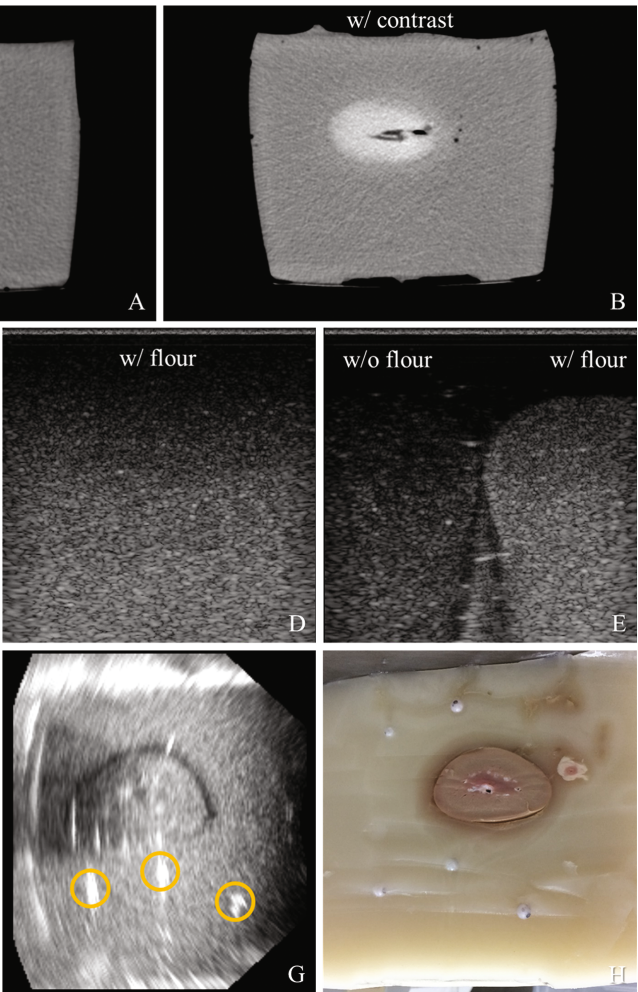

Fig. 3. CT images of phantoms (A) without and (B) with contrast agent. US images of the TMM (C) without and (D) with flour. (E) Both TMM side-by-side. The same FM visualized in (F) CT and $(\mathrm{G})$ US images. The same FM are marked with a yellow circle. $(\mathrm{H})$ Section of the phantom with implanted FM. 
Additionally, Fig. 3 shows the aspect of FM on the CT and US images. As expected, it is possible to observe that polystyrene material has a different contrast on CT and US. Besides, it is possible to observe an equal distribution of the FM on both image modalities (Fig. 3F-G).

\subsection{Fiducial Registration Error and Volume Differences}

Seventeen of the twenty-four correspondent FM were manually selected on both CT and US volumes. One point on the surface of each fiducial marker was selected manually. The RMS error was $1.1 \mathrm{~mm}$. Visual inspection of the registration revealed a coherent overlap between structures and FM (Fig. 4).

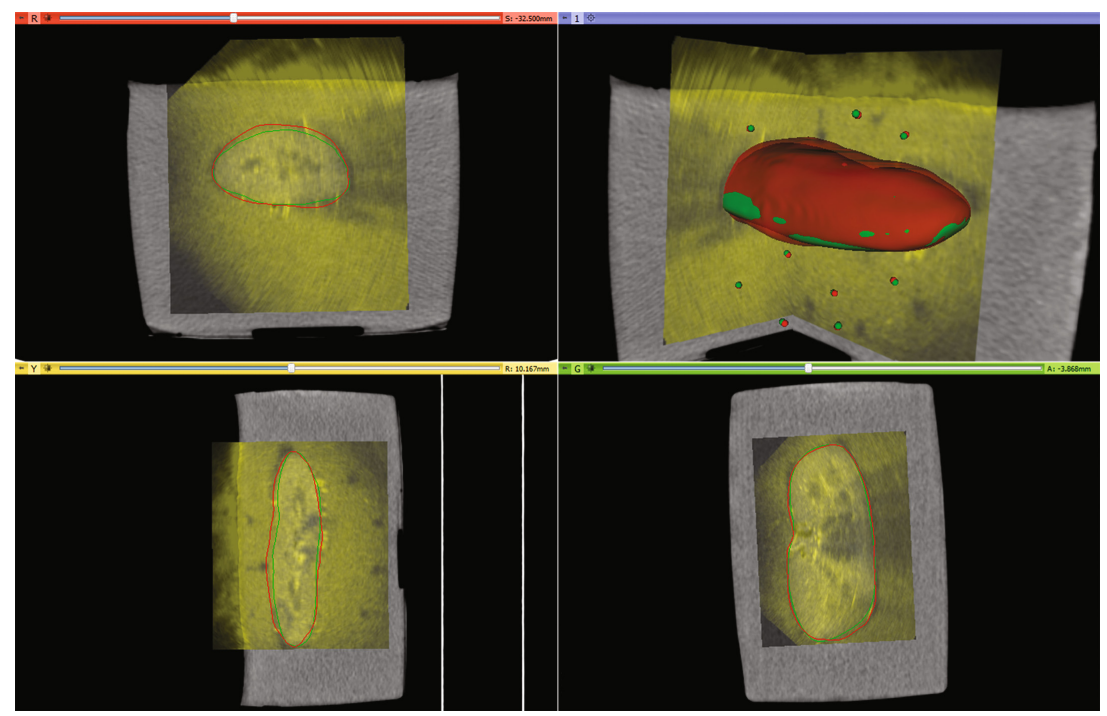

Fig. 4. Images and segmented surfaces aligned based on selected FM from both CT and US. CT images, surfaces, and FM are represented by gray level images, green surfaces and points, respectively, while US by yellow level images, red surfaces and points.

Regarding segmentation, CT and US manual contours presented small differences (Fig. 4). The CT images presented a surface area of $213 \mathrm{~cm}^{2}$ and volume of $203 \mathrm{ml}$, while the US images obtained a surface area of $229 \mathrm{~cm}^{2}$ and volume of $238 \mathrm{ml}$.

\section{Discussion}

We presented a protocol to construct a kidney phantom model made of the porcine kidney, the TMM and implanted FM. The initial results are promising since the kidney is completely visible on both CT and US images. Despite ex-vivo tissues have a quicker degradation than artificial materials, their use shows promising results mainly 
due to the realistic images obtained. Relevant anatomical boundaries and some anatomical details are detected. Nonetheless, the renal cortex is usually more hypoechoic in human US images [23]. Overall, the TMM meets the requirements, which is essential, mainly, to obtain US images. Moreover, FM are visible and can be easily targeted, which is extremely important for the registration validation stage. These results strongly suggest that this phantom model can be used to image-guided PRA since a valid ground-truth was achieved using realistic images. Thus, these models may lead to stronger eIGI methods before going forward to in-vivo validation. In addition, this phantom model has potential to be used for medical teaching and training. Specifically, it is possible to previously evaluate the kidney on CT images and, then, perform the PRA using intraoperative imaging.

Although the appearance of the CT images was slightly different, the contrast agent enhanced the kidney tissues with respect to the background, which is a common characteristic in the non-contrast images. Indeed, without contrast agent, TMM and the kidney presented the same Hounsfield values, hampering their differentiation.

We believe that the manufacturing still presents some drawbacks, namely the presence of small air bubbles within the TMM and near the FM. Indeed, this may disrupt the perception of the FM on US images, with its correction potentially improving the sphere visualization and reducing the current fiducial registration error of $1.1 \mathrm{~mm}$. However, the high number and non-linear (but known) distribution of the FM tends to minimize the errors caused by the air bubbles and any intrinsic image alignment errors [21]. Moreover, it must be taken into account that the image resolution and even some image artifacts inherently influence the method's accuracy.

Surface and volume differences were observed between both US- and CT-based results. These are in part explained by the expected inter-modality variability throughout the segmentation stage. This fact must be addressed in a future study.

This work was strongly influenced by the work developed by Xiang et al., where the authors used a porcine phantom model to study the registration of 3D US to CT angiography of the kidney [13]. We presented some modifications to their protocol, namely a different mixture of TMM, number and position of FM, and construction method. These modifications bring relevant advantages for image-guided PRA since: (1) flour enhances speckle pattern around the kidney on the US images; (2) higher number and distributed FM allow image acquisition with different transducer's positions and orientations without losing performance; (3) pouring the TMM solution at once reduces the number of undesirable interfaces on the US images. Nevertheless, we would like to emphasize that some factors on both protocols can change the kidney tissue properties, like the TMM temperature throughout the pouring and cooling stages that may change the natural tissue contrast and echogenicity in both CT and US images. These factors may be addressed in a future work.

Finally, as future work, we intend to introduce motion and common anatomical artifacts, as recently presented by Lee et al. [24] for high-intensity focused US. 


\section{Conclusion}

We manufactured a kidney phantom model for image-guided PRA for dual modal CT and US imaging. Herewith, we obtained similar preoperative and intraoperative data of common MIKI and a valid ground-truth with enough accuracy to assess errors, which can be used to develop new methods for image-guided PRA.

eIGI in the abdominal region is still a challenging task, due to the deformable nature and motion of the tissues. Despite this phantom does not consider these parameters, this work is a step forward on building a validation tool for image-guided PRA.

Acknowledgments. The authors would like to thank Paulo Marques, Bruno Oliveira, Paulo Mota, Emanuel Carvalho Dias, Manuela Carneiro, Fernando Veloso and to the departments of urology and imagiology of Hospital de Braga for their contribution to this work.

Funding. This work has been funded by FEDER funds, through the Competitiveness Factors Operational Programme (COMPETE), and by National funds, through the Foundation for Science and Technology (FCT), under the scope of the project NORTE-01-0145-FEDER-000013, supported by the NORTE 2020, under the Portugal 2020 Partnership Agreement, through the European Regional Development Fund (FEDER). J. Gomes-Fonseca, A. Miranda, P. Morais, and S. Queirós were funded by FCT under the Ph.D. grants PD/BDE/113597/2015, SFRH/BD/52059/ 2012, SFRH/BD/95438/2013, and SFRH/BD/93443/2013, respectively.

\section{References}

1. Marcovich, R., Smith, A.D.: Percutaneous renal access: tips and tricks. BJU Int. 95(s2), 78-84 (2005)

2. Knoll, T., Michel, M.S., Alken, P.: Surgical atlas percutaneous nephrolithotomy: the Mannheim technique. BJU Int. 99(1), 213-231 (2007)

3. de la Rosette, J.J.M.C.H., Laguna, M.P., Rassweiler, J.J., Conort, P.: Training in percutaneous nephrolithotomy-a critical review. Eur. Urol. 54(5), 994-1001 (2008)

4. de la Rosette, J., Assimos, D., Desai, M., Gutierrez, J., Lingeman, J., Scarpa, R., Tefekli, A.: The clinical research office of the endourological society percutaneous nephrolithotomy global study: indications, complications, and outcomes in 5803 patients. J. Endourol. 25(1), 11-17 (2011)

5. Rodrigues, P.L., Rodrigues, N.F., Fonseca, J., Lima, E., Vilaça, J.L.: Kidney targeting and puncturing during percutaneous nephrolithotomy: recent advances and future perspectives. J. Endourol. 27(7), 826-834 (2013)

6. Cleary, K., Peters, T.M.: Image-guided interventions: technology review and clinical applications. Annu. Rev. Biomed. Eng. 12(1), 119-142 (2010)

7. Li, X., Long, Q., Chen, X., He, D., Dalin, H., He, H.: Real-time ultrasound-guided PCNL using a novel SonixGPS needle tracking system. Urolithiasis 42(4), 341-346 (2014)

8. Ungi, T., Beiko, D., Fuoco, M., King, F., Holden, M.S., Fichtinger, G., Siemens, D.R.: Tracked ultrasonography snapshots enhance needle guidance for percutaneous renal access: a pilot study. J. Endourol. 28(9), 1040-1045 (2014) 
9. Rodrigues, P.L., Vilaça, J.L., Oliveira, C., Cicione, A., Rassweiler, J., Fonseca, J., Rodrigues, N.F., Correia-Pinto, J., Lima, E.: Collecting system percutaneous access using real-time tracking sensors: first pig model in vivo experience. J. Urol. 190(5), 1932-1937 (2013)

10. Lima, E., Rodrigues, P.L., Mota, P., Carvalho, N., Dias, E., Correia-Pinto, J., Autorino, R., Vilaça, J.L.: Ureteroscopy-assisted percutaneous kidney access made easy: first clinical experience with a novel navigation system using electromagnetic guidance (IDEAL stage 1). Eur. Urol. 30, 214-226 (2017)

11. Leroy, A., Mozer, P., Payan, Y., Troccaz, J.: Rigid registration of freehand 3D ultrasound and CT-scan kidney images. In: Medical Image Computing and Computer-Assisted Intervention - MICCAI 2004: 7th International Conference, Saint-Malo, France, September 26-29, 2004. Proceedings, Part I, pp. 837-844 (2004)

12. Li, Z.-C., Li, K., Zhan, H.-L., Chen, K., Gu, J., Wang, L.: Augmenting intraoperative ultrasound with preoperative magnetic resonance planning models for percutaneous renal access. Biomed. Eng. Online 11(1), 60-76 (2012)

13. Xiang, J., Gill, S., Nguan, C.Y., Abolmaesumi, P., Rohling, R.N.: Registration of ultrasound to CT angiography of kidneys: a porcine phantom study. In: Proceedings of SPIE 7625, vol. 7625, p. 762518-8 (2010)

14. Hunt, A., Ristolainen, A., Ross, P., Öpik, R., Krumme, A., Kruusmaa, M.: Low cost anatomically realistic renal biopsy phantoms for interventional radiology trainees. Eur. J. Radiol. 82(4), 594-600 (2013)

15. Ristolainen, A., Ross, P., Gavšin, J., Semjonov, E., Kruusmaa, M.: Economically affordable anatomical kidney phantom with calyxes for puncture and drainage training in interventional urology and radiology. Acta Radiol. Short Rep. 3(5), 2047981614534231 (2014)

16. Adams, F., Qiu, T., Mark, A., Fritz, B., Kramer, L., Schlager, D., Wetterauer, U., Miernik, A., Fischer, P.: Soft 3D-printed phantom of the human kidney with collecting system. Ann. Biomed. Eng. 45(4), 963-972 (2017)

17. Thompson, S.M., Ramirez-Giraldo, J.C., Knudsen, B., Grande, J.P., Christner, J.A., Xu, M., Woodrum, D.A., McCollough, C.H., Callstrom, M.R.: Porcine ex vivo liver phantom for dynamic contrast-enhanced computed tomography: development and initial results. Invest. Radiol. 46(9), 586-593 (2011)

18. DeSimio, T., Baron, T.: New ex-vivo porcine model for endoscopic ultrasound-guided training in transmural puncture and drainage of pancreatic cysts and fluid collections (with videos). Endosc. Ultrasound 4(1), 34 (2015)

19. Teirlinck, C.J., Bezemer, R.A., Kollmann, C., Lubbers, J., Hoskins, P.R., Ramnarine, K.V., Fish, P., Fredeldt, K.E., Schaarschmidt, U.G.: Development of an example flow test object and comparison of five of these test objects, constructed in various laboratories. Ultrasonics 36(1-5), 653-660 (1998)

20. Earle, M., De Portu, G., DeVos, E.: Agar ultrasound phantoms for low-cost training without refrigeration. Afr. J. Emerg. Med. 6(1), 18-23 (2016)

21. Bao, N., Chen, Y., Yue, Y., Li, H., Cui, Z., Zhuang, J., Tian, S., Kang, Y.: Fiducial markers configuration optimization in image-guided surgery. Biomed. Mater. Eng. 24(6), 3361-3371 (2014)

22. Horn, B.K.P.: Closed-form solution of absolute orientation using unit quaternions. J. Opt. Soc. Am. A 4(4), 629 (1987)

23. Hansen, K.L., Nielsen, M.B., Ewertsen, C.: Ultrasonography of the kidney: a pictorial review. Diagnostics (Basel, Switzerland) 6(1), 2 (2015)

24. Lee, D., Koizumi, N., Tsukihara, H., Azuma, T., Nomiya, A., Yoshinaka, K., Sugita, N., Homma, Y., Matsumoto, Y., Mitsuishi, M.: Construction of kidney phantom model with acoustic shadow by rib bones and respiratory organ motion, p. 150007 (2017) 